\title{
Article \\ Whole-Genome Sequencing Improves the Diagnosis of DFNB1 Monoallelic Patients
}

\author{
Anaïs Le Nabec ${ }^{1, * D}$, Mégane Collobert ${ }^{1}\left(\mathbb{D}\right.$, Cédric Le Maréchal ${ }^{1,2}$, Rémi Marianowski ${ }^{3}$, Claude Férec $^{1,2}$ \\ and Stéphanie Moisan 1,2,*D \\ 1 Univ Brest, Inserm, EFS, UMR 1078, GGB, F-29200 Brest, France; megane_collobert@hotmail.fr (M.C.); \\ cedric.lemarechal@univ-brest.fr (C.L.M.); claude.ferec@gmail.com (C.F.) \\ 2 Laboratoire de Génétique Moléculaire et d'Histocompatibilité, CHRU Brest, UMR 1078, F-29200 Brest, France \\ 3 Service ORL et Chirurgie Cervicofaciale du CHRU Brest, F-29200 Brest, France; \\ remi.marianowski@chu-brest.fr \\ * Correspondence: lenabec.anais@gmail.com (A.L.N.); moisan.stephanie@hotmail.fr (S.M.); \\ Tel.: +33-2-98-01-65-84 (A.L.N.); +33-2-98-01-65-67 (S.M.)
}

check for updates

Citation: Le Nabec, A.; Collobert, M.; Le Maréchal, C.; Marianowski, R.; Férec, C.; Moisan, S. Whole-Genome Sequencing Improves the Diagnosis of DFNB1 Monoallelic Patients. Genes 2021, 12, 1267. https://doi.org/ $10.3390 /$ genes 12081267

Academic Editor: Michele Cioffi

Received: 3 August 2021

Accepted: 17 August 2021

Published: 19 August 2021

Publisher's Note: MDPI stays neutral with regard to jurisdictional claims in published maps and institutional affiliations.

Copyright: (c) 2021 by the authors. Licensee MDPI, Basel, Switzerland. This article is an open access article distributed under the terms and conditions of the Creative Commons Attribution (CC BY) license (https:// creativecommons.org/licenses/by/ $4.0 /)$.

\begin{abstract}
Hearing loss is the most common sensory defect, due in most cases to a genetic origin. Variants in the GJB2 gene are responsible for up to $30 \%$ of non-syndromic hearing loss. Today, several deafness genotypes remain incomplete, confronting us with a diagnostic deadlock. In this study, whole-genome sequencing (WGS) was performed on 10 DFNB1 patients with incomplete genotypes. New variations on GJB2 were identified for four patients. Functional assays were realized to explore the function of one of them in the GJB2 promoter and confirm its impact on GJB2 expression. Thus, in this study WGS resolved patient genotypes, thus unlocking diagnosis. WGS afforded progress and bridged some gaps in our research.
\end{abstract}

Keywords: WGS; DFNB1; deafness; variants; functional assays; cCRE; non-coding variation

\section{Introduction}

Hearing loss is the most common sensory pathology, affecting about 1-2 in every 1000 newborns, with a prevalence which increases with age [1-4]. In industrialized countries, congenital deafness has a genetic origin in $80 \%$ of cases [5]. Deafness can be syndromic or not (associated or not with other pathologies or malformations), respectively representing 10\% and $90 \%$ of cases. More than 500 syndromes are associated with syndromic deafness and more than 100 genes have been described in non-syndromic hearing loss (NSHL) (Van Camp G, Smith RJH. Hereditary Hearing Loss Homepage. https:/ / hereditaryhearingloss.org, the 10 May 2021).

NSHL can be classified according to heredity. Generally $80-90 \%$ of those affected have autosomal recessive inheritance (DFNB), $10-15 \%$ have a dominant mode (DFNA), $1 \%$ of cases are associated with the $\mathrm{X}$ chromosome (DFNX), and others have a mitochondrial inheritance mode $[5,6]$.

The predominant form is autosomal recessive non-syndromic hearing loss (DFNB1). Most DFNB1 phenotypes are described as prelingual and bilateral non-syndromic hearing loss, this being severe to profound. This type of deafness affects all frequencies and is not associated with inner ear malformations. Vestibular function remains unaffected $[1-5,7,8]$. The GJB2 (Gap Junction $\beta$ 2-chr13:20,187,470-20,192,938 (hg38)) gene is mainly implicated in DFNB1 with frequencies ranging from $20 \%$ to $40 \%$, according to populations with the most frequent mutation, c.35delG [3,5,9-11].

Moreover, seven large DFNB1 deletions have been described in DFNB1 patients: del-920 kb [12], del-101 kb del(GJB2-D13S175) [13], del(GJB6-D13S1830) [14], del(GJB6D13S1854) [15], del-131kb [16], del-179kb [17], and del-8kb [18]. This year, Brozkova et al. described a DFNB1 deletion of $3 \mathrm{~kb}$ in one patient [11]. 
The genomic architecture of our chromosomes is now far better investigated and understood. Many studies have focused on the role of non-coding regions and on genetic variants they contain, opening up new research possibilities. However, a vast majority of coding and non-coding variants may remain of unknown clinical significance [19-21].

Almost $8 \%$ of the human genome is covered with candidate cis-regulatory elements (cCREs) [22]. The identification of distal acting regulatory elements has been the object of active research in recent years. Disruptions of such regulatory elements and/or chromatin conformation are likely to play a critical role in human genetic diseases [19-21].

Routine molecular diagnosis in the Molecular Genetics Laboratory at Brest University Hospital involves the testing of around 80 deaf patients each year, and among these patients, $\sim 20 \%$ are DFNB1 biallelic carriers. However, several genotypes remain incomplete; for monoallelic DFNB1, which represents fewer than $1 \%$ of the tested patients, most patients are carriers of the c.35delG heterozygous and some have rare variants.

The c.35delG heterozygous genotype may be related to the general population frequency with an overall frequency of $2 \%$ but an excess of the deletion has been shown in the deaf population [8].

To accelerate a patient's diagnosis odyssey, we propose the investigation of non-coding DNA such as GJB2 CREs. Exploration of structural variations which could disrupt DFNB1 $3 \mathrm{D}$ regulating looping model will be important. Moreover, variations in other deafness genes could explain phenotypes.

To resolve these cases, whole-genome sequencing (WGS) was performed on 10 monoallelic DFNB1 carriers of rare variants. In this study, WGS resolved some genotypes, thus unlocking diagnoses. Moreover, we identified a new mutation in the GJB2 promoter which impacts GJB2 gene regulation.

\section{Materials and Methods}

\subsection{Ethics Statement}

All patients gave their informed consent for inclusion before they participated in the study. The study was conducted in accordance with the Declaration of Helsinki (1975), and the protocol was approved by the Ethics Committee of Brest (Protocol N $\left.{ }^{\circ} 29 \mathrm{BRC} 19.0104\right)$.

\subsection{Recruitment of Patient/Population}

Ten monoallelic DFNB1 patients with non-syndromic bilateral, stable, mild to profound deafness were included in this study after routine molecular diagnosis at the Molecular Genetics Laboratory at Brest University Hospital between 1990 and 2020. Eight patients with rare variant were diagnosed, including one patient (patient P5) with del(GJB6D13S1830), and one c.35delG family case (patient P4). All patients had permanent hearing loss, not associated with infection or drugs. For these patients, CGH array genotyping had already been performed, with no deletion or duplication at the DFNB1 locus detected. Before WGS, Sanger sequencing had been realized to screen exon 2 of the GJB2 gene.

\subsection{Whole Genome Sequencing}

\subsubsection{Laboratory}

DNA was submitted for whole-genome sequencing (Integragen Genomics platform). PCR free libraries were prepared with NEBNext Ultra II DNA Library Prep Kits according to supplier recommendations. Specific double-strand gDNA quantification and a fragmentation (300 ng of input with high-molecular-weight gDNA) sonication method were used to obtain approximately $400 \mathrm{bp}$ fragments. Finally, paired-end adaptor oligonucleotides (xGen TS-LT Adapter Duplexes from IDT) were ligated and re-paired. Tailed fragments were purified for direct sequencing without a PCR step.

DNA PCR free libraries were sequenced on paired-end $150 \mathrm{pb}$ runs on the Illumina NovaSeq apparatus. Image analysis and base calling were performed using Illumina Real Time Analysis (RTA) Pipeline version 3.4.4 with default parameters. 


\subsubsection{Bioinformatics}

Sequence reads were mapped on the Human Genome Build (hg38) with the BurrowsWheeler Aligner (BWA) tool [23].

Integragen Genomics uses Broad Institute's GATK Haplotype Caller GVCF tool (GATK 3.8.1) [24] for single nucleotide variations (SNV) and small insertions and deletions.

Variants were annotated with Ensembl's VEP (Variant Effect Predictor, release VEP 95.1) [25] (which takes data available in gnomAD, the 1000 Genomes Project, Kaviar Databases ... ) by Integragen Genomics. Then, 5 algorithms (DANN, FATHMM, MutationTaster, SIFT and Polyphen) were used to predict pathogenicity of single nucleotide polymorphism (SNP) [26-28].

\subsection{Data Analysis}

\subsubsection{SNV Identification}

Different analyses were performed: all deafness genes were annotated and variations with a frequency of less than 0.1 were analyzed (215 genes in Supplementary Material-Table S1). Variations in all genes with a frequency of less $0.07 \%$ were also studied in public databases (1000 Genomes Project, GnomAD, Kaviar) [29].

\subsubsection{SV Identification}

Integragen Genomics used different algorithms to investigate structural variations: Lumpy (v 0.2.13), Delly (v 0.7.9) and Manta (v 1.5.0) on bam files.

The BreakDancer algorithm was also used to investigate structural variations.

\subsection{Confirmation and Segregation Analysis}

Variant Confirmation

Each candidate variant was confirmed using Sanger sequencing on ABI3130XL (Thermo Fisher Scientific Inc., Waltham, MA, USA) using the Big Dye Terminator Cycle Sequencing V3.1 Ready Reaction Kit (Life Technologies, Carlsbad, CA, USA). Segregations were performed when the DNA of parents was available.

\subsection{Functional Assays Cells}

\subsubsection{Plasmid Constructs}

All the cloning steps were done using the "In fusion ${ }^{\circledR "}$ strategy from Clontech. Using the pGL3-Basic Vector (Promega), the $5^{\prime}$-flanking region of the GJB2 gene (1541 bp, " $\mathrm{P}_{G J B 2}$ ") was cloned upstream from the firefly luciferase cDNA at the Hind III site. CREs and combinations were amplified and inserted downstream. All the inserted fragments were verified by sequencing. The PCR primers used to amplify the GJB2 promoter and CREs are given in Supplementary Material-Table S2.

\subsubsection{Mutagenesis}

Mutagenesis was performed using a QuickChange II XL Site-Directed Mutagenesis kit from Agilent Technologies. The primers are presented in Supplementary Material-Table S3.

\subsubsection{Luciferase Assays}

For luciferase assays, $1.25 \times 10^{5}$ cells (SAEC: Small Airways Epithelial cells) were seeded in 12-well plates. Transfections were undertaken $24 \mathrm{~h}$ later with the transit 2020 reagent (Mirus). Here, $400 \mathrm{ng}$ of the $\mathrm{P}_{G J B 2}$ constructs and $100 \mathrm{ng}$ of a pCMV-LacZ construct (as an internal control) were used for each condition. Every condition was used in triplicate. At $48 \mathrm{~h}$ post-transfection the cells were washed once with $1 \times$ PBS and lysed with Passive lysis buffer (Promega). Cells lysates were clarified by centrifugation at $12,000 \times g$ for $5 \mathrm{~min}$ at $4{ }^{\circ} \mathrm{C}$. Then, $20 \mu \mathrm{L}$ of each protein extract was used to assay the luciferase activity and $25 \mu \mathrm{L}$ for $\beta$-galactosidase activity. We used Promega reagents and the Varioscan multiwell plate reader (Thermo Fisher). Results are presented as relative luciferase activity, 
with the $\mathrm{P}_{G J B 2}$ construct activity equal to 1 . The significance of the increased luciferase activity was determined using analysis of variance and $t$-tests with $R$.

\section{Results}

In order to detect an unknown causal variant, WGS was performed on 10 monoallelic DFNB1 patients. This study involved nine patients who carried one GJB2 heterozygous mutation (Table 1) and one patient (Patient P5) who carried the heterozygous deletion del(GJB6-D13S1830).

\section{1. $S N V$}

\subsubsection{GJB2 Mutations}

WGS analysis identified a second mutation on the GJB2 gene in patients P3, P4, P8, and P10 (Table 2).

In the routine molecular diagnosis, the Sanger sequencing of the GJB2 gene of patient P3 allowed us to detect frameshift variation (rs730880338) at c.269. Then, a missense variation (rs80338945) was discovered by WGS analysis at the same position. Indeed, these variations in the same nucleotide complicated the interpretation of Sanger sequencing analysis. The duplication hid the other mutation so that the initial Sanger sequencing interpretation failed to detect the two mutations.

DNA samples from parents were not available for segregation analysis, but singlestrand NGS sequencing (see IGV (Integrative Genome Viewer) BAM visualization (Figure 1) confirmed that these variants are in trans.
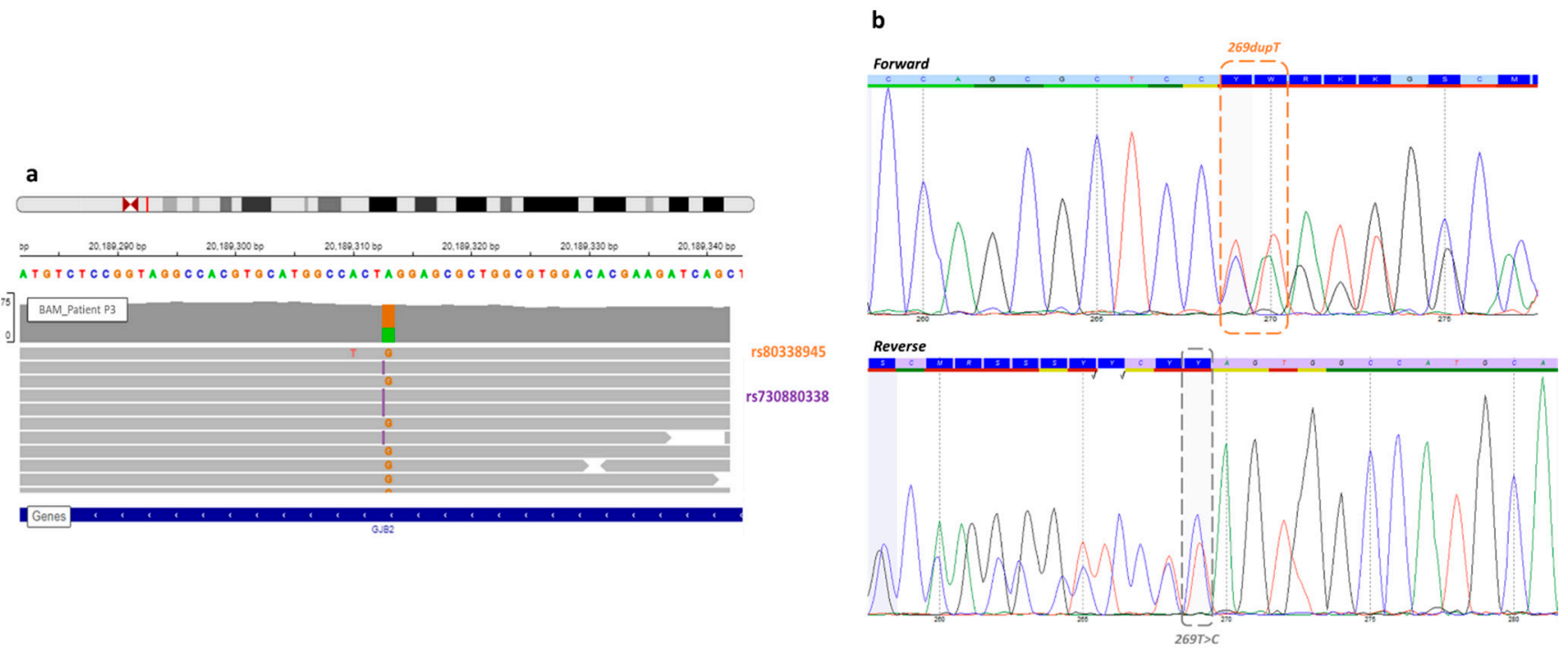

Figure 1. View of the missense variant and frameshift GJB2 gene of patient P3. (a) In the Integrative Genome Viewer, the frameshift variant (rs730880338) at c.269 GJB2 position, known before WGS (orange), and the missense variant (rs80338945) discovered by WGS analysis (purple). Each variant was on a different read, so this analysis confirmed a trans configuration. (b) A new Sanger sequencing in forward and reverse detected both mutations, but it still remains difficult to interpret.

Patient 4 carried two GJB2 mutations, a recurrent mutation, in deaf population, only the c.35delG (rs8033893) have been detected by DHPLC (Denaturing High Performance Liquid Chromatography). The second mutation discovered by WGS analysis was a frameshift (rs730880338), c.269dup (Figure 2). Indeed, since no hetero- and homo-duplex was detected by HPLC we did not realize Sanger sequencing. This is why genotype was unresolved before WGS. Variant segregation of parents allowed the determination of variants transmission for their children (Figure 3). 
Table 1. Patient genotypes (monoallelic) at the DFNB1 locus before WGS

\begin{tabular}{|c|c|c|c|c|c|c|c|c|c|c|c|c|}
\hline Patient & $\begin{array}{l}\text { Deafness } \\
\text { Phenotype }\end{array}$ & Case & Gene & HGVSc & Chr. & $\begin{array}{l}\text { Position } \\
\text { (hg38) }\end{array}$ & HGVSp & Impact & Consequences & $\begin{array}{l}\text { Variant } \\
\text { Class }\end{array}$ & rs Number & $\begin{array}{c}\text { Allele } \\
\text { Frequency } \\
\text { (GnomAD) }\end{array}$ \\
\hline P1 & Profound & Single & GJB2 & NM_004004.5:c.132G > A & chr13 & 20189450 & NP_003995.2:p.Trp44Ter & HIGH & stop_gained & SNV & rs104894407 & 0.00001470 \\
\hline P3 & Profound & Single & GJB2 & NM_004004.5:c.269dup & chr13 & 20189312 & NP_003995.2:p.Val91SerfsTer11 & HIGH & frameshift_variant & insertion & rs730880338 & 0.00002940 \\
\hline P4 & Profound & Family & GJB2 & NM_004004.5:c.35del & chr13 & 20189546 & NP_003995.2:p.Gly12ValfsTer2 & HIGH & frameshift_variant & deletion & rs80338939 & 0.009802 \\
\hline P5 & Profound & Single & & & & & $\operatorname{del}(G J B 6-D 13 S 1830)$ & & & & & 0.0003935 \\
\hline P7 & Profound & Single & GJB2 & NM_004004.5:c.633T > A & chr13 & 20188949 & NP_003995.2:p.Cys211Ter & HIGH & stop_gained & SNV & - & - \\
\hline P8 & Profound & Family & GJB2 & NM_004004.5:c.139G > T & chr13 & 20189443 & NP_003995.2:p.Glu47Ter & HIGH & stop_gained & SNV & rs104894398 & 0.0001176 \\
\hline P9 & Profound & Single & GJB2 & NM_004004.5:c.313_326del & chr13 & 20189255 & NP_003995.2:p.Lys105GlyfsTer5 & HIGH & frameshift_variant & deletion & rs111033253 & 0.0003234 \\
\hline P10 & Mild & Single & GJB2 & NM_004004.5:c.139G > T & chr13 & 20189443 & NP_003995.2:p.Glu47Ter & HIGH & stop_gained & SNV & rs104894398 & 0.0001176 \\
\hline
\end{tabular}

Table 2. DFNB1 mutations detected by WGS.

\begin{tabular}{|c|c|c|c|c|c|c|c|c|c|c|}
\hline Patient & Gene & HGVSc & Chr. & $\begin{array}{c}\text { Position } \\
\text { (hg38) }\end{array}$ & HGVSp & Impact & Consequences & Variant Class & rs Number & $\begin{array}{c}\text { Allele } \\
\text { Frequency } \\
\text { (GnomAD) }\end{array}$ \\
\hline \multirow{2}{*}{ P3 } & GJB2 & NM_004004.5:c.269dup & chr13 & 20189312 & NP_003995.2:p.Val91SerfsTer11 & HIGH & frameshift_variant & insertion & rs730880338 & 0.00002940 \\
\hline & GJB2 & NM_004004.5:c.269T >C & chr13 & 20189313 & NP_003995.2:p.Leu90Pro & MODERATE & missense_variant & SNV & rs80338945 & 0.001161 \\
\hline \multirow{2}{*}{ P4 } & GJB2 & NM_004004.5:c.35del & chr13 & 20189546 & NP_003995.2:p.Gly12ValfsTer2 & HIGH & frameshift_variant & deletion & rs80338939 & 0.009802 \\
\hline & GJB2 & NM_004004.5:c.269dup & chr13 & 20189312 & NP_003995.2:p.Val91SerfsTer11 & HIGH & frameshift_variant & insertion & rs730880338 & 0.00002940 \\
\hline \multirow{2}{*}{ P8 } & GJB2 & NM_004004.5:c.139G > T & chr13 & 20189443 & NP_003995.2:p.Glu47Ter & HIGH & stop_gained & SNV & rs104894398 & 0.0001176 \\
\hline & GJB2 & NM_004004.5:c.-23 + 1G > A & chr13 & 20192782 & & HIGH & splice_donor_variant & SNV & rs80338940 & 0.0003236 \\
\hline \multirow[t]{2}{*}{ P10 } & GJB2 & $\mathrm{G}>\mathrm{C}$ & chr13 & 20193022 & 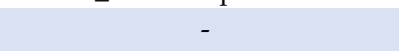 & UNKNOWN & upstream_gene_variant & SNV & rs1425012952 & 0.00001472 \\
\hline & GJB2 & $\mathrm{G}>\mathrm{T}$ & chr13 & 20183294 & - & UNKNOWN & downstream_gene_variant & $\mathrm{SNV}$ & rs372782198 & 0.003099 \\
\hline
\end{tabular}


a

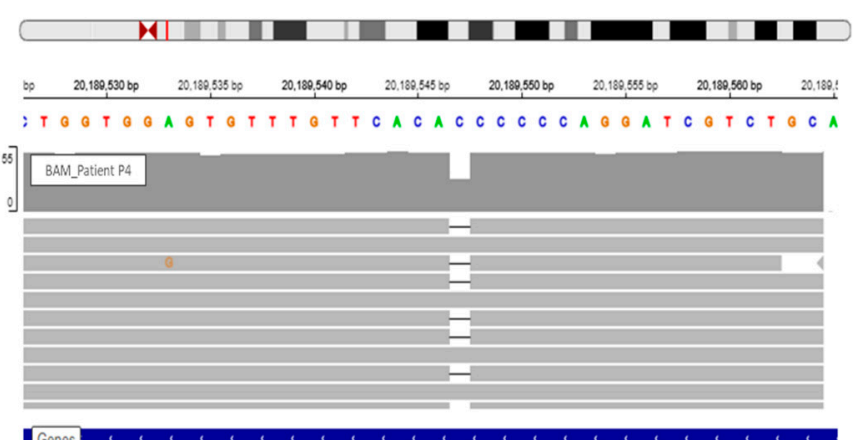

b

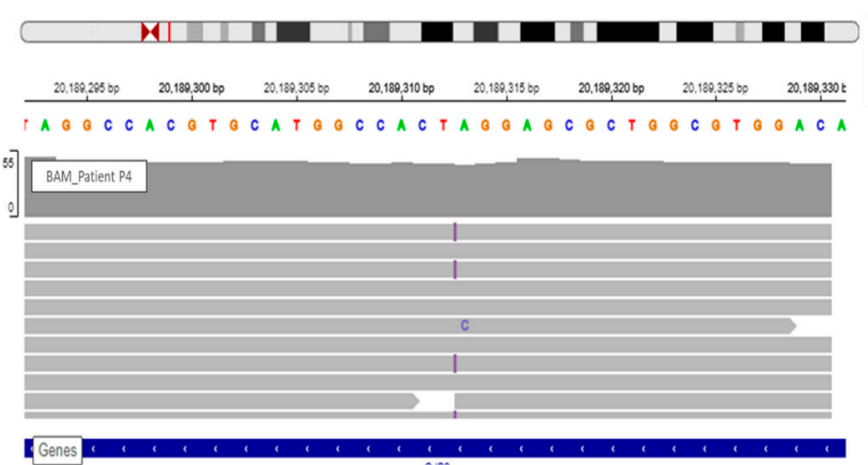

Q.82

Figure 2. View of GJB2 variants of patient P4 in IGV. (a) WGS confirmed the known c.35delG. (b) The frameshift variation, c.269dup, detected via WGS and observed in IGV.

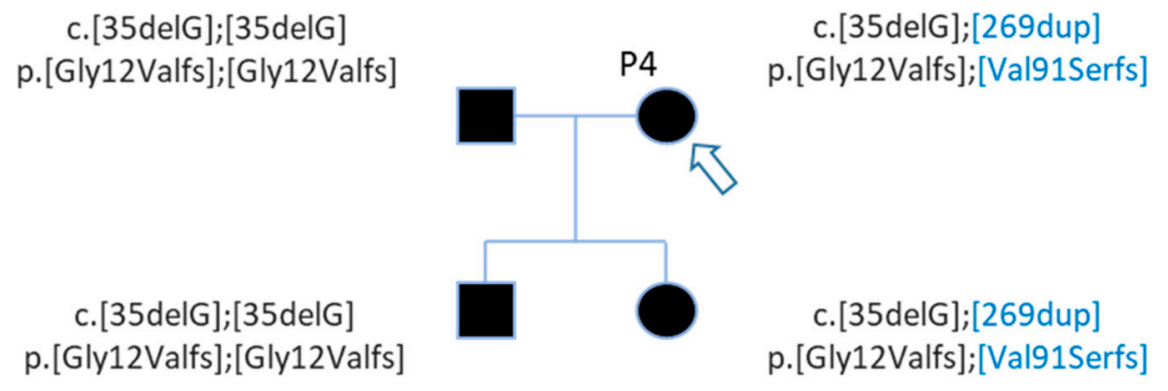

Figure 3. Pedigree of patient P4 with 2 GJB2 variants. Patient P4 (arrow) carries two GJB2 mutations, the c.35delG known before WGS and the c.269dup discovered by WGS analysis (in blue). WGS analysis detected mutations for her daughter also.

The first Sanger sequencing of Patient P8 was performed in 2002; only the nonsense mutation of GJB2 was detected (rs104894398-c.139G > T) (Figure 4).

a
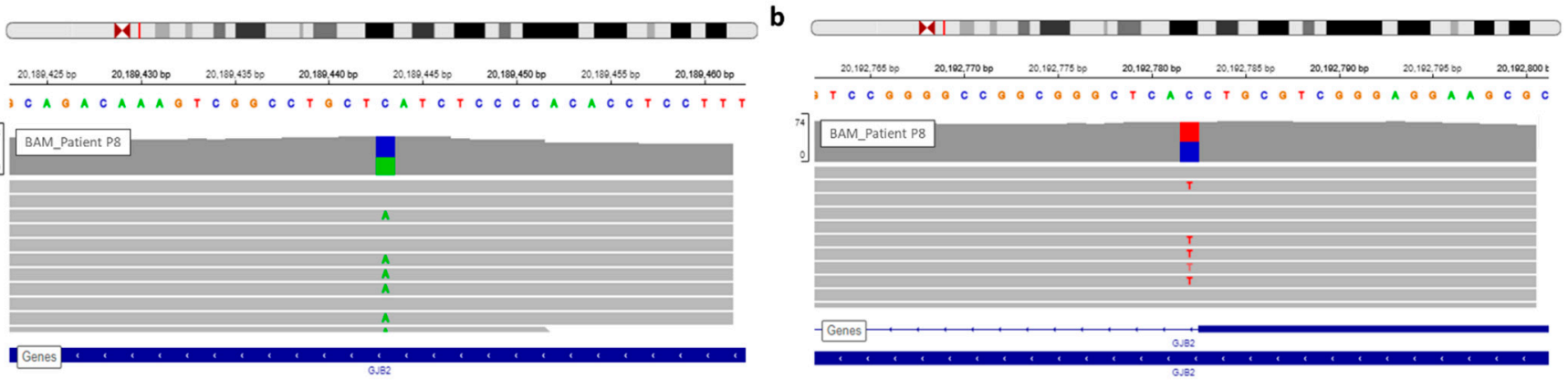

Figure 4. View of GJB2 variants of patient P8 in IGV. (a) The nonsense variation, c.139G > T, discovered during routine care. (b) The second GJB2 variation detected by WGS analysis is a splice site mutation.

WGS analysis allowed identification of a second GJB2 mutation, a splice site mutation (rs80338940—c.-23 + 1G > A) on intron 1 of the GJB2 gene (Figure 4). This variant had not been found earlier because the sequencing of exon 1 has only been routinely done in the laboratory since 2005.

After WGS, Sanger sequencing confirmed this second mutation, and segregation confirmed that these mutations are in trans (Figure 5). The genotypes of patient P8 and her brother are c.[-23 + 1G > A];[139G > T] p.[?];[Glu47Ter] (Figure 5). 


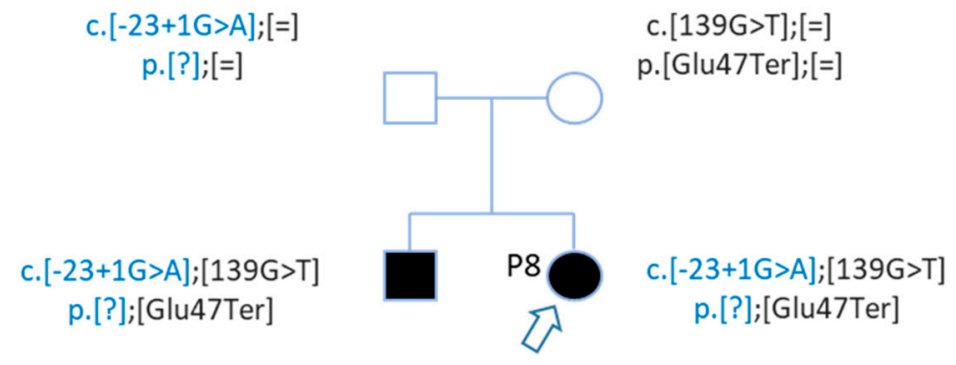

Figure 5. Pedigree of patient P8 with 2 GJB2 mutations in trans. Patient P8 and her brother carried 2 mutations in trans. Mutations were inherited from each parent.

\subsubsection{GJB2 Upstream Variation}

Patient P10 carried one nonsense GJB2 mutation (c.139G > A;p.Glu47Ter). WGS analysis allowed us to identify two variants on GJB2: rs1425012952 and rs372782198. These variants were respectively upstream and downstream of GJB2. The upstream variant was located on the GJB2 promoter, particularly on a GC box located at -81 bp of the TSS (Transcription Start Site) (Figure 6). This GC box has been described as useful and critical for GJB2 basal transcription with the binding of Sp1 transcription factor [20]. This variant is reported once in GnomAD, with one carrier.

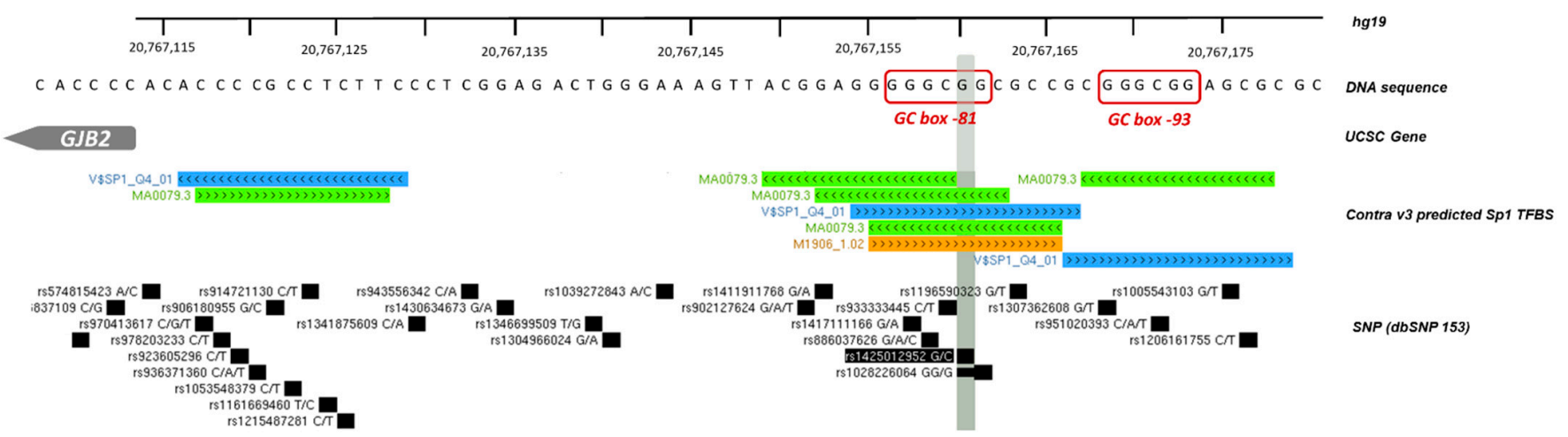

Figure 6. GJB2 promoter and upstream variants detected via WGS analysis. The GJB2 promoter has 2 GC boxes (each located respectively at -81 and $-93 \mathrm{bp}$ of TSS) that are useful for GJB2 basal transcription. The upstream GJB2 variant is located on GC box -81 and Contrat v3 predicts several Sp1 binding sites at this location (Bioinformatic tool to predict transcription factor binding sites http:/ / bioit2.irc.ugent.be/contra/v3/\#/step/1, the 21 May 2021).

Sanger analysis of parents confirmed a trans segregation (Figure 7), indeed the mother was carrying this upstream GJB2 variant, and the father the nonsense mutation. The upstream GJB2 variant was not detected during routine molecular diagnosis the first time, because the sequencing concerned only exons and intron of GJB2.

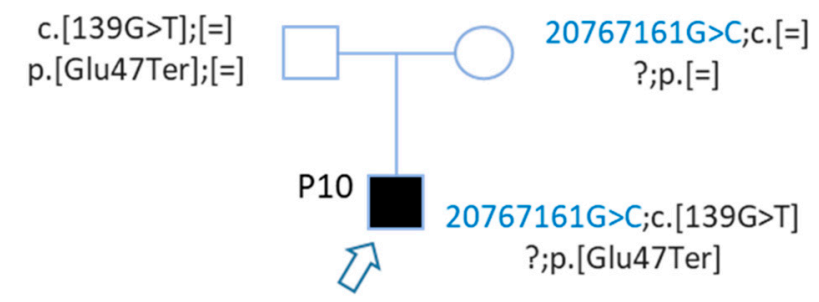

Figure 7. Segregation of GJB2 variants of patient P10. Hearing parents were carrying each a GJB2 variant, the nonsense and the upstream variant. Patient P10 carried these 2 mutations in trans.

Patient P10 carried these two mutations, a nonsense and an upstream variant, in trans, which may explain his deafness. To understand, the impact of GJB2 upstream variant on GJB2 gene expression, functional in-vitro assays were performed. Indeed, Contra v3 
(in silico this is to predict transcription factor binding sites (TFBSs)) predicted several Sp1 TFBSs just at the location of this upstream variant (Figure 6). Moreover, in 1997, Kiang and et al., described GJB2 regulation by binding of Sp1 transcription factor in the GC box -81 .

Functional assays allowed us to analyze the GJB2 promoter variant on GJB2 gene expression by firefly luciferase expression following transient transfection into cells. For this, different constructions are realized, one with the WT GJB2 promoter $\left(\mathrm{P}_{G J B 2}\right)$, one with the insertion of this variant on the GJB2 promoter $\left(\mathrm{P}_{G J B 2} \Delta\right)$. Moreover, in our previous paper, the C3 region (chr13:20419404-20420446 (hg38)-1043 pb) was described for the first time as the most important GJB2 enhancer [30]. Thus, we also decided to study the impact of the upstream GJB2 variant on GJB2 expression with and without the $\mathrm{C} 3$ enhancer.

$\mathrm{P}_{G J B 2}$ WT construction has an expression equal to 1 and $\mathrm{P}_{G J B 2} \Delta$ construction significantly decreased the GJB2 expression (decrease of $17 \%$ ). Moreover, the GJB2 promoter with $\mathrm{C} 3$ enhancer $\left(\mathrm{P}_{G J B 2} \mathrm{WT}+\mathrm{C} 3\right)$ showed an GJB2 expression of 7 but this construction with the SNP promoter $\left(\mathrm{P}_{G J B 2} \Delta+\mathrm{C} 3\right)$ exhibited a two-fold decrease in GJB2 expression. (Figure 8).

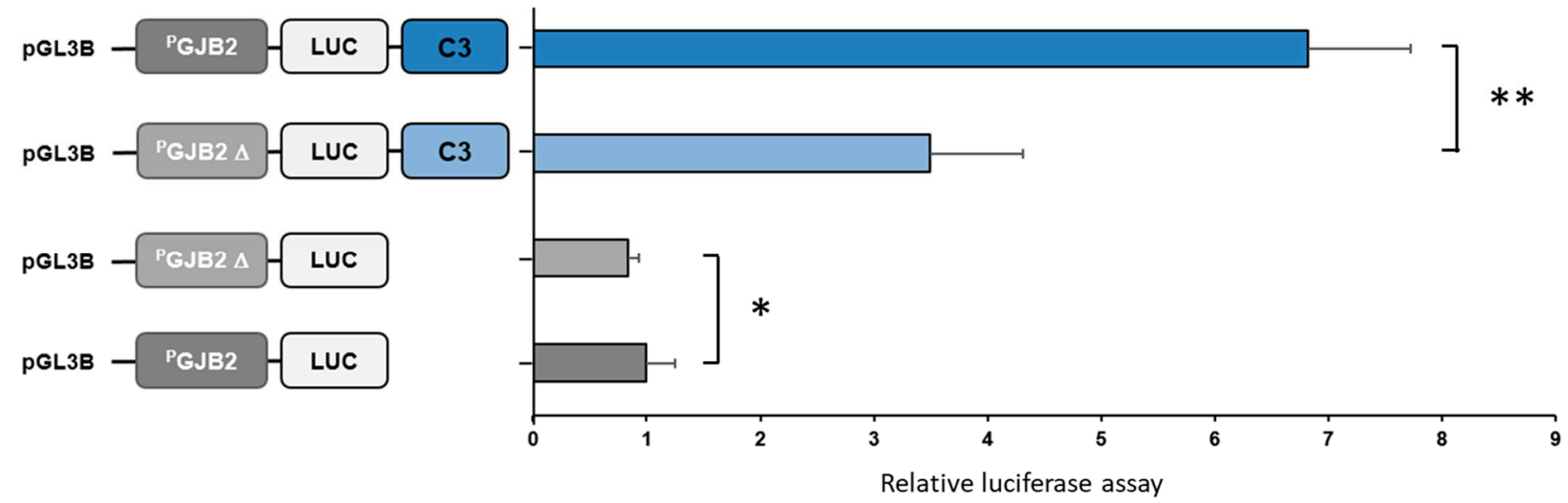

Figure 8. Functional assays of GJB2 promoter variant. Luciferase reporter constructs with the GJB2 WT promoter ( $\mathrm{P}_{G J B 2}$; $1043 \mathrm{bp}), \mathrm{GJB} 2$ mutated promoter $\left(\mathrm{P}_{\mathrm{GJB} 2} \Delta ; 1043 \mathrm{bp}\right)$, and constructions with $\mathrm{C} 3$ enhancer were transfected in SAEC cells. ${ }^{*} p<0.05 ;{ }^{* *} p<1.33 \times 10^{-7}$ using analysis of variance and $t$-tests.

\subsubsection{SNVs on Deafness Genes}

For the five other patients carrying one nonsense mutation, no mutation was identified on GJB2, or on CREs of the GJB2 gene and DFNB1 locus. For this reason, we screened genes linked to deafness to identify potential pathogenic variations. Heterozygous pathogenic or likely pathogenic variants (single nucleotide, indel, or copy number variants) were detected in known NSHL genes in five probands but they were not sufficient to explain the phenotype.

\section{USH1C Gene}

Patient P5 was the only patient to carry a DFNB1 deletion: del(GJB6-D13S1830). This deletion of $309 \mathrm{~kb}$ removed CRYL1 and GJB6 genes but GJB2 remained intact (Figure 9). To explore any possibilities, we screened other deafness genes.

Among deafness genes, three variants have been identified on the USH1C gene: a nonsense variation (rs377145777), a missense variant (rs1064074), and a splice site variation with unknown impact on protein (Table 3). Parental DNA were not available to study segregation. 


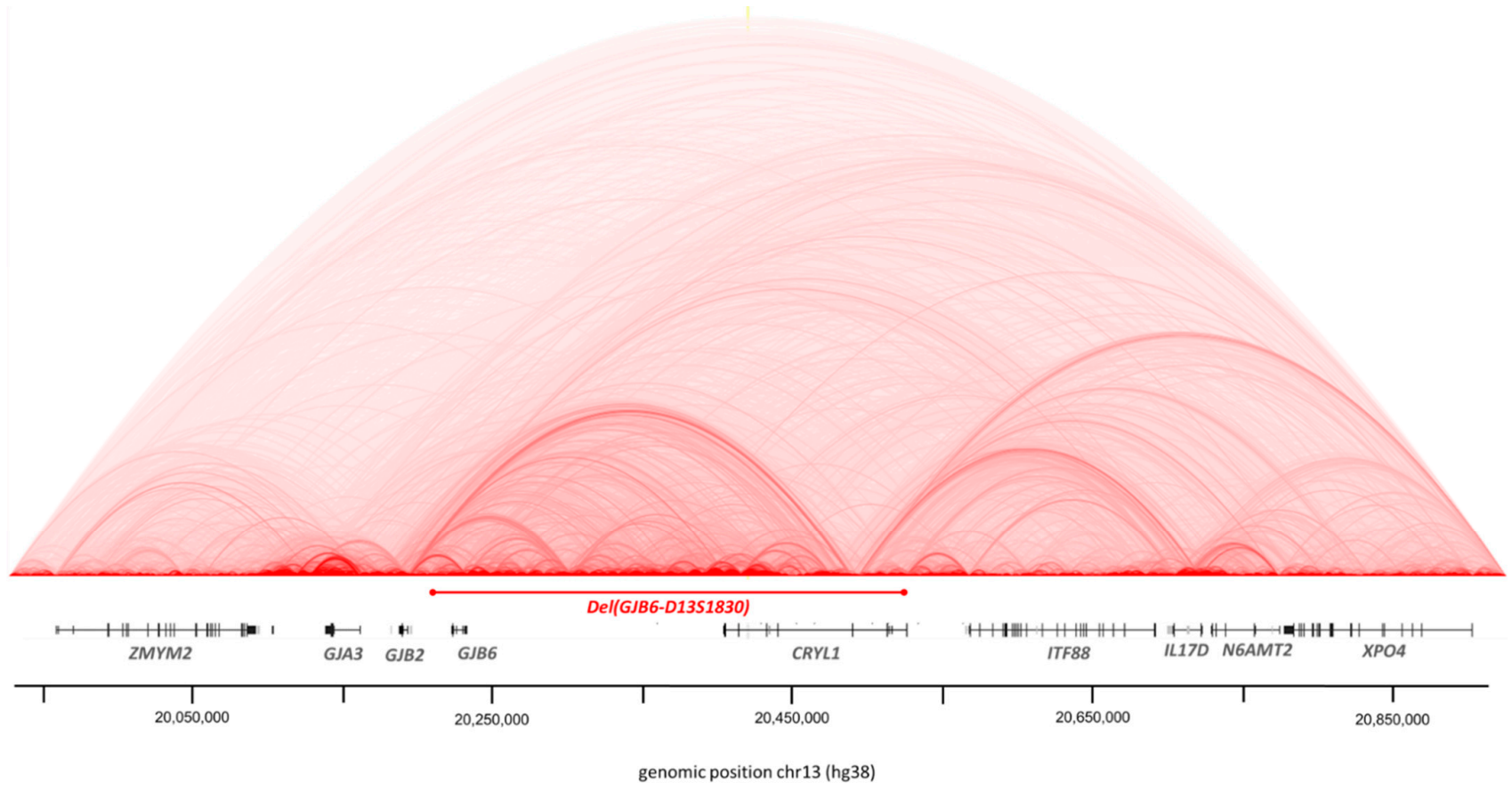

Figure 9. Deletion del(GJB6-D13S1830) of patient P5. Deletion del(GJB6-D13S1830) disrupted GJB6 gene and CRYL1 gene but GJB2 remained intact.

Table 3. USH1C variants of patient P5. Three variants detected via WGS analysis.

\begin{tabular}{|c|c|c|c|c|c|c|c|c|c|}
\hline Gene & HGVSc & Chr. & $\begin{array}{l}\text { Position } \\
\text { (hg38) }\end{array}$ & $\begin{array}{l}\text { Protein } \\
\text { Variation }\end{array}$ & Impact & Consequences & $\begin{array}{l}\text { Variant } \\
\text { Class }\end{array}$ & rs Number & $\begin{array}{c}\text { Allele } \\
\text { Frequency } \\
\text { (GnomAD) }\end{array}$ \\
\hline USH1C & NM_001297764.1:c.463C > T & chr11 & 17527256 & $\mathrm{R} 155^{*}$ & HIGH & stop_gained & SNV & rs377145777 & 0.00001759 \\
\hline USH1C & $\begin{array}{c}\text { NM_001297764.1:c.1589+ } \\
\text { 3_1589 + 6del }\end{array}$ & chr11 & 17496751 & $\ldots$ & LOW & $\begin{array}{c}\text { splice_region_variant\&in- } \\
\text { tron_variant }\end{array}$ & deletion & - & - \\
\hline USH1C & NM_005709.3:c.1557G > C & chr11 & 17498195 & E519D & MODERATE & missense_variant & SNV & rs1064074 & 0.5511 \\
\hline
\end{tabular}

\subsection{Structural Variations}

Structural variations (SVs) corresponded to chromosomic rearrangements such as deletion, insertion, duplication, and translocation which could disrupt one or several loci, genes, or regulatory regions at the same time.

\subsubsection{CNV by Integragen Genomics}

Integragen Genomics proposed a CNV (Copy Number Variation) study for all patients. This included only deletion and duplication variations. These analyses allowed us to confirm the deletion del(GJB6-D13S1830) of patient P5 (Figure 9). No other variant on other regions of the DFNB1 has been detected by this tool for the other patients.

\subsubsection{BreakDancer Algorithm for SVs}

To continue SV analysis, BreakDancer algorithm was used on all samples. The algorithm brought to light a few hundred variations by chromosome. We focused on chromosome 13 at the DFNB1 locus implicated on NSHL.

For patients with unresolved genotypes, some SVs were detected on chromosome 13, around the DFNB1 locus. These SV do not disrupt either the GJB2 gene or the DFNB1 locus but we do not know their impact on other genes. 


\section{Discussion}

Hearing loss is the main sensorial deficit in developed countries, with more than $80 \%$ of cases being of genetic origin. Although more than 100 genes have been identified in non-syndromic deafness, for certain patients no genotypes nor environmental causes can be identified [5].

The key message of this work is the impact of WGS to improve molecular base knowledge of NSHL.

WGS analysis resolved genotypes for four patients. Indeed, for three patients, genotypes have been elucidated with a second mutation on GJB2 gene. Indeed, for these patients the second event in GJB2 has not been detected in routine care. Although, Whole Exome Sequencing (WES) could have been detected these variants on GJB2 (as c.269T > C variant (P3), c.269dup (P4) c.-23 + 1G > A (P8)), WES would not have detected GJB2 promoter variant (P10). Here, WGS allowed for the study of non-coding and intronic DNA besides coding sequences. Thus, WGS allows the detection of small deletion in the non-coding regions of the genome as well as some inversion that is not detected by Exome sequencing. Moreover, the cost of WGS is close to the cost of WES, and will probably decrease in the next years.

For one patient, two variants were present at the same nucleotide in trans, yielding the Sanger sequencing interpretation complex. WGS allowed for easier identification by separation of the two alleles.

Another patient carried a nonsense mutation and a splice mutation in GJB2 (patient P3). In routine care in 2000s, splice sites were not screened, and this splice variation (c.-23 + 1G > A) was described for the first time in 2002 [1]. This can explain why we discovered this mutation by WGS analysis. Since 2005, splice sites have been screened in all patients in routine care. Since this case, we screened the splice sites on all DNA from patients before 2005, and another patient with this variant was detected.

For patient P4, the second event (c.269dup) was not detected by DHPLC. Indeed, in the 2000s, for the first screening, DHPLC was used to detect nucleotide alterations in PCR products without sequencing. When a mutation was detected by DHPLC, it was confirmed by Sanger sequencing. However, for this case, DHPLC did not detect the c.269dup variant showing a technical deficit.

After these WGS results, all DFNB1 monoallelic patients analyzed with DHPLC were screened by Sanger sequencing.

Moreover, for several years, we were only interested in $2 \%$ of the coding regions of the genome, the exome [19]. However, recent studies interesting by non-coding DNA, showed that $8 \%$ of genome correspond to cCRE regions [22]. Discovery and interpretation of these cCREs are important because they play important roles on gene expression, chromatin organization at different cell states [22].

Many publications have highlighted defects in chromatin organization and the disruption of regulatory regions leading to pathologies, and many publications describe defects in embryonic development or cancers [31-33]. Therefore, it is important to consider the non-coding genome and non-coding variations.

Few publications describe GJB2 gene regulation, at the proximal or distal level. Human GJB2 promoter has been described for the first time in 1997 by Kiang and his collaborators [34]. The following year, promoter and basal regions essential for GJB2 expression have been characterized [35]. Following Wilch's publication hypothesized that some deafness could result from disruption of GJB2 cis-regulatory elements (CREs) [16,36], we were the first to explore GJB2 cis-regulation. We described several CREs of GJB2 and suggested a 3D DFNB1 regulation locus model [30].

Indeed, with this paper, we bring to light the impact of one non-coding variant. Indeed, one patient carries a promoter variation which has an impact on GJB2 expression. $G J B 2$ expression is reduced and when this variant is present in conjunction with the GJB2 enhancer, GJB2 expression is reduced to $50 \%$. Each allele of patient P10 carries a GJB2 mutation, the nonsense mutation produces a connexin 26 truncated protein and the 
upstream variation decreases GJB2 expression. This patient presents mild deafness, which can be explained by GJB2 transcripts reduced by promoter variants, but not completely.

This is the second mutation reported to affect the GJB2 promoter. Matos et al., found a g.-77C > T variant in the compound heterozygous state with mutation p.(Val84Met) in a Portuguese deaf patient. This variant is also in the GC box at $-81 \mathrm{pb}$ of TSS and alters transcription [37].

After these results, we screened 25 monoallelic other DFNB1 patients (rare variant or c.35delG), but none of them presented this promoter variant. It is most likely a private variant.

Moreover, this upstream variation may explain deafness phenotypes (degree of hearing loss) [38,39]. Indeed, if GJB2 expression is altered, the connexin 26 level is modified and modulates the phenotype.

Some cases of presbycusis emerge more prematurely, maybe it is due to decrease of GJB2 expression by a cis-regulatory variants and correlates with environment factors.

These results demonstrate the necessity to investigate the non-coding genome to overcome wandering and diagnostic deadlock with investigation of chromatin conformation and regulatory variants. Functional assays will be necessary to explore the function of non-coding regions and validate new genes or variants of uncertain significance implicated in genetic diseases.

Cis-acting regions may be a therapeutic challenge that could lead to the development of specific molecules capable of modulating gene expression in the future. A better understanding of regulatory mechanisms of gene expression could elucidate cases of patients where the phenotype is not yet explained by the genotype. This would thus help in better diagnosis and therefore better management. The analysis of cis-regulatory regions will allow the functional impact exploration of certain genomic rearrangements or variants in the chromatin organization. When genomic rearrangements or SNVs are located in non-coding regions, a study of the three-dimensional organization of chromatin study and an analysis of the TADs (Topologically associated domains) in the loci possibly involved in genetic diseases will be proposed by chromatin conformation approaches derived from 3C (Chromosome Conformation Capture). Functional assays (gene reporter tests, chromatin immunoprecipitation and CRISPR-Cas9 analysis) could be used to validate potential regulatory variants.

Six genotypes remained unresolved, although the DFNB1 locus has been screened along with other deafness genes. This may be explained by the fact that the genome hg38 was not complete, some regions are hidden as repeat sequences or telomeric regions. However, new human reference genome described 2226 paralogous gene copies, whose 115 are predicted to be protein coding, and cover repeat sequences but their function is unknown $[40,41]$. Perhaps it is not surprising that we cannot resolve all genotypes if we do not have all the information.

Structural variations analysis did not provide answers. Upstream WGS and CGH arrays were performed on DFNB1 locus for all patients to detect DFNB1 deletions or duplications. However, CGH arrays hide repeated sequences. Thus, these sequences are excluded. Moreover, CGH arrays cannot identify unbalanced rearrangements. After WGS and SV analysis we hoped to detect unbalanced rearrangements, but the bioinformatic tool, BreakDancer, used to analyze structural variations did not highlight causal variations at the DFNB1 locus $[42,43]$. WGS produces a lot of data, but SV detection algorithms have strengths and weaknesses, and some algorithms do not allow the identification of all types of SV $[42,44]$. We could perhaps discuss whether we did not detect SVs because patients did not have SVs or whether this was due to technology limitations.

Although the DFNB1 locus was screened, we do not have explanations for these patients. There are some limitations in methodologies which remove repeated sequences or non-aligned reads; it would be interesting to use other techniques such as PacBio Sequencing to have long reads. 
Through this project, we aim to better understand molecular mechanisms and we expect to reduce the diagnostic odyssey. Ensuring a definitive diagnosis will have a huge impact on patients who have spent years in a diagnostic deadlock, receiving multiple misdiagnoses resulting in inappropriate treatments.

Furthermore, every test, procedure, treatment, on a misdiagnosis amounts to wasteful spending in healthcare.

Thus, patients and families should receive appropriate therapy with a good genetic counselling, and be well-managed and advised in their daily lives.

\section{Conclusions}

In conclusion, WGS allowed us to correct the genotypes of patients with NSHL by rectifying routine care Sanger sequencing.

We resolved 4 genotypes out of 10 by identifying a second event on the GJB2 gene, including a promoter variant. Indeed, WGS identified a non-coding variation in the GJB2 promoter, with an impact on GJB2 expression.

Six genotypes remain unresolved, amongst which one is possibly due to another deafness gene (USH1C).

In this day and age, WGS is an essential tool, as illustrated well in this work, and we propose that it is today to best strategy for improving knowledge for deafness patients.

Supplementary Materials: The following are available online at https:/ /www.mdpi.com/article/10 .3390/genes12081267/s1, Table S1: 215 deafness genes screened; Table S2: PCR primer sequences used for cloning into the luciferase reporter construct $\left(5^{\prime}-3^{\prime}\right)$ Table S3: PCR primer sequences used for mutagenesis $\left(5^{\prime}-3^{\prime}\right)$.

Author Contributions: Conceptualization, A.L.N. and S.M.; methodology, A.L.N.; software, A.L.N.; validation, A.L.N., S.M., and C.L.M.; formal analysis, A.L.N.; resources, A.L.N., S.M.; data curation, A.L.N.; writing—original draft preparation, A.L.N., S.M.; writing—review and editing, A.L.N., M.C., S.M., C.L.M., R.M., C.F.; visualization, A.L.N.; supervision, S.M., C.L.M., R.M., C.F.; project administration, S.M.; funding acquisition, A.L.N., S.M., C.F. All authors have read and agreed to the published version of the manuscript.

Funding: This research was funded by French foundations: "La Fondation pour 1'Audition" $\left(\mathrm{N}^{\circ}\right.$ FPARD-2018-9), "Région Bretagne", the association "Gaétan Salaün" and the PHRC ( ${ }^{\circ}$ R0803, 2005).

Institutional Review Board Statement: The study was conducted according to the guidelines of the Declaration of Helsinki, and was approved by the Ethics Committee of Brest (Protocol $\mathrm{N}^{\circ}$ 29BRC19.0104).

Informed Consent Statement: All patients gave their informed consent for inclusion before they participated in the study.

Data Availability Statement: Data are available upon request from the authors.

Acknowledgments: We thank the French foundation "La Fondation pour l'Audition", the "Région Bretagne" and the association "Gaétan Salaün" for supporting the project. We thank Kévin Uguen, Thomas Ludwig for their help with bioinformatics tools. We also thank Anthony Herzig for grammatical/editorial English corrections of this manuscript.

Conflicts of Interest: The authors declare that they have no conflict of interest.

\section{References}

1. Denoyelle, F.; Marlin, S.; Weil, D.; Moatti, L.; Chauvin, P.; Garabédian, É.-N.; Petit, C. Clinical features of the prevalent form of childhood deafness. Lancet 1999, 353, 1298-1303. [CrossRef]

2. Feldmann, D.; Denoyelle, F.; Chauvin, P.; Garabédian, E.-N.; Couderc, R.; Odent, S.; Joannard, A.; Schmerber, S.; Delobel, B.; Leman, J.; et al. Large deletion of the GJB6 gene in deaf patients heterozygous for the GJB2 gene mutation: Genotypic and phenotypic analysis. Am. J. Med. Genet. Part A 2004, 127, 263-267. [CrossRef] 
3. Marlin, S.; Feldmann, D.; Blons, H.; Loundon, N.; Rouillon, I.; Albert, S.; Chauvin, P.; Garabédian, E.-N.; Couderc, R.; Odent, S.; et al. GJB2 and GJB6 Mutations: Genotypic and Phenotypic Correlations in a Large Cohort of Hearing-Impaired Patients. Arch. Otolaryngol. Head Neck Surg. 2005, 131, 481-487. [CrossRef] [PubMed]

4. Morton, C.C.; Nance, W.E. Newborn Hearing Screening-A Silent Revolution. N. Engl. J. Med. 2006, 354, 2151-2164. [CrossRef] [PubMed]

5. Tanaka-Ouyang, L.; Marlin, S.; Nevoux, J. Les surdités d'origine génétique. Press. Med. 2017, 46, 1089-1096. [CrossRef]

6. Dror, A.A.; Avraham, K.B. Hearing impairment: A panoply of genes and functions. Neuron 2010, 68, 293-308. [CrossRef] [PubMed]

7. Gandía, M.; del Castillo, F.J.; Rodríguez-Álvarez, F.J.; Garrido, G.; Villamar, M.; Calderón, M.; Moreno-Pelayo, M.A.; Moreno, F.; del Castillo, I. A Novel Splice-Site Mutation in the GJB2 Gene Causing Mild Postlingual Hearing Impairment. PLoS ONE 2013, 8, e0073566. [CrossRef]

8. Leclère, J.-C.; Le Gac, M.-S.; Le Maréchal, C.; Ferec, C.; Marianowski, R. GJB2 mutations: Genotypic and phenotypic correlation in a cohort of 690 hearing-impaired patients, toward a new mutation? Int. J. Pediatr. Otorhinolaryngol. 2017, 102, 80-85. [CrossRef]

9. Roux, A.-F.; Pallares-Ruiz, N.; Vielle, A.; Faugère, V.; Templin, C.; Leprevost, D.; Artières, F.; Lina, G.; Molinari, N.; Blanchet, P.; et al. Molecular epidemiology of DFNB1 deafness in France. BMC Med. Genet. 2004, 5, 5. [CrossRef]

10. Kenneson, A.; Van Naarden Braun, K.; Boyle, C. GJB2 (connexin 26) variants and nonsyndromic sensorineural hearing loss: A HuGE review. Genet. Med. 2002, 4, 258-274. [CrossRef]

11. Brozkova, D.S.; Meszarosova, A.U.; Lassuthova, P.; Varga, L.; Staněk, D.; Borecká, S.; Laštůvková, J.; Čejnová, V.; Rašková, D.; Lhota, F.; et al. The Cause of Hereditary Hearing Loss in GJB2 Heterozygotes-A Comprehensive Study of the GJB2/DFNB1 Region. Genes 2021, 12, 684. [CrossRef]

12. Feldmann, D.; Le Maréchal, C.; Jonard, L.; Thierry, P.; Czajka, C.; Couderc, R.; Ferec, C.; Denoyelle, F.; Marlin, S.; Fellmann, F. A new large deletion in the DFNB1 locus causes nonsyndromic hearing loss. Eur. J. Med. Genet. 2009, 52, 195-200. [CrossRef]

13. Bliznetz, E.A.; Lalayants, M.R.; Markova, T.G.; Balanovsky, O.P.; Balanovska, E.V.; Skhalyakho, R.A.; Pocheshkhova, E.A.; Nikitina, N.V.; Voronin, S.V.; Kudryashova, E.K.; et al. Update of the GJB2/DFNB1 mutation spectrum in Russia: A founder Ingush mutation del(GJB2-D13S175) is the most frequent among other large deletions. J. Hum. Genet. 2017, 62, 789-795. [CrossRef]

14. Lerer, I.; Sagi, M.; Ben-Neriah, Z.; Wang, T.; Levi, H.; Abeliovich, D. A deletion mutation in GJB6 cooperating with a GJB2 mutation in trans in non-syndromic deafness: A novel founder mutation in Ashkenazi Jews. Hum. Mutat. 2001, 18, 460. [CrossRef] [PubMed]

15. del Castillo, F.J.; Rodríguez-Ballesteros, M.; Alvarez, A.; Hutchin, T.; Leonardi, E.; de Oliveira, C.A.; Azaiez, H.; Brownstein, Z.; Avenarius, M.R.; Marlin, S.; et al. A novel deletion involving the connexin-30 gene, del(GJB6-d13s1854), found in trans with mutations in the GJB2 gene (connexin-26) in subjects with DFNB1 non-syndromic hearing impairment. J. Med. Genet. 2005, 42, 588-594. [CrossRef] [PubMed]

16. Wilch, E.; Azaiez, H.; Fisher, R.A.; Elfenbein, J.; Murgia, A.; Birkenhäger, R.; Bolz, H.; Da Silva-Costa, S.M.; Del Castillo, I.; Haaf, T.; et al. A novel DFNB1 deletion allele supports the existence of a distant cis-regulatory region that controls GJB2 and GJB6 expression. Clin. Genet. 2010, 78, 267-274. [CrossRef] [PubMed]

17. Tayoun, A.N.A.; Mason-Suares, H.; Frisella, A.L.; Bowser, M.; Duffy, E.; Mahanta, L.; Funke, B.; Rehm, H.L.; Amr, S.S. Targeted Droplet-Digital PCR as a Tool for Novel Deletion Discovery at the DFNB1 Locus. Hum. Mutat. 2015, 37, 119-126. [CrossRef]

18. Abe, S.; Nishio, S.; Yokota, Y.; Moteki, H.; Kumakawa, K.; Usami, S. Diagnostic pitfalls for GJB2-related hearing loss: A novel deletion detected by Array-CGH analysis in a Japanese patient with congenital profound hearing loss. Clin. Case Rep. 2018, 6, 2111-2116. [CrossRef]

19. Gasperini, M.; Tome, J.M.; Shendure, J. Towards a comprehensive catalogue of validated and target-linked human enhancers. Nat. Rev. 2020, 21, 292-310. [CrossRef]

20. Epstein, D.J. Cis-regulatory mutations in human disease. Brief. Funct. Genom. Proteom. 2009, 8, 310-316. [CrossRef]

21. Kleinjan, D.-J.; Coutinho, P. Cis-ruption mechanisms: Disruption of cis-regulatory control as a cause of human genetic disease. Brief. Funct. Genom. Proteom. 2009, 8, 317-332. [CrossRef] [PubMed]

22. Abascal, F.; Acosta, R.; Addleman, N.J.; Adrian, J.; Afzal, V.; Aken, B.; Akiyama, J.A.; Jammal, O.A.; Amrhein, H.; Anderson, S.M.; et al. Expanded encyclopaedias of DNA elements in the human and mouse genomes. Nature 2020, 583, 699-710. [CrossRef]

23. Li, H.; Durbin, R. Fast and accurate short read alignment with Burrows-Wheeler transform. Bioinformatics 2009, 25, 1754-1760. [CrossRef]

24. Poplin, R.; Ruano-Rubio, V.; DePristo, M.A.; Fennell, T.J.; Carneiro, M.O.; Van der Auwera, G.A.; Kling, D.E.; Gauthier, L.D.; Levy-Moonshine, A.; Roazen, D.; et al. Scaling accurate genetic variant discovery to tens of thousands of samples. bioRxiv 2018, 201178. [CrossRef]

25. McLaren, W.; Gil, L.; Hunt, S.E.; Riat, H.S.; Ritchie, G.R.S.; Thormann, A.; Flicek, P.; Cunningham, F. The Ensembl Variant Effect Predictor. Genome Biol. 2016, 17, 112. [CrossRef]

26. Quang, D.; Chen, Y.; Xie, X. DANN: A deep learning approach for annotating the pathogenicity of genetic variants. Bioinformatics 2015, 31, 761. [CrossRef]

27. Shihab, H.A.; Gough, J.; Cooper, D.N.; Stenson, P.D.; Barker, G.L.A.; Edwards, K.J.; Day, I.N.M.; Gaunt, T.R. Predicting the Functional, Molecular, and Phenotypic Consequences of Amino Acid Substitutions using Hidden Markov Models. Hum. Mutat. 2013, 34, 57-65. [CrossRef] 
28. Schwarz, J.M.; Cooper, D.N.; Schuelke, M.; Seelow, D. MutationTaster2: Mutation prediction for the deep-sequencing age. Nat. Methods 2014, 11, 361-362. [CrossRef]

29. Oza, A.M.; DiStefano, M.T.; Hemphill, S.E.; Cushman, B.J.; Grant, A.R.; Siegert, R.K.; Shen, J.; Chapin, A.; Boczek, N.J.; Schimmenti, L.A.; et al. Expert specification of the ACMG/AMP variant interpretation guidelines for genetic hearing loss. Hum. Mutat. 2018, 39, 1593-1613. [CrossRef]

30. Moisan, S.; Le Nabec, A.; Quillévéré, A.; Le Maréchal, C.; Férec, C. Characterization of GJB2 cis-regulatory elements in the DFNB1 locus. Hum. Genet. 2019, 138, 1275-1286. [CrossRef]

31. Spielmann, M.; Lupiáñez, D.G.; Mundlos, S. Structural variation in the 3D genome. Nat. Rev. Genet. 2018, 19, 453-467. [CrossRef]

32. Lupiáñez, D.G.; Kraft, K.; Heinrich, V.; Krawitz, P.; Brancati, F.; Klopocki, E.; Horn, D.; Kayserili, H.; Opitz, J.M.; Laxova, R.; et al. Disruptions of topological chromatin domains cause pathogenic rewiring of gene-enhancer interactions. Cell 2015, 161, 1012-1025. [CrossRef] [PubMed]

33. Northcott, P.A.; Lee, C.; Zichner, T.; Stütz, A.M.; Erkek, S.; Kawauchi, D.; Shih, D.J.H.; Hovestadt, V.; Zapatka, M.; Sturm, D.; et al. Enhancer hijacking activates GFI1 family oncogenes in medulloblastoma. Nature 2014, 511, 428-434. [CrossRef]

34. Kiang, D.T.; Jin, N.; Tu, Z.J.; Lin, H.H. Upstream genomic sequence of the human connexin 26 gene. Gene 1997, $199,165-171$. [CrossRef]

35. Tu, Z.J.; Kiang, D.T. Mapping and characterization of the basal promoter of the human connexin26 gene. Biochim. Biophys. Acta 1998, 1443, 169-181. [CrossRef]

36. Wilch, E.; Zhu, M.; Burkhart, K.B.; Regier, M.; Elfenbein, J.L.; Fisher, R.A.; Friderici, K.H. Expression of GJB2 and GJB6 is reduced in a novel DFNB1 allele. Am. J. Hum. Genet. 2006, 79, 174-179. [CrossRef]

37. Matos, T.D.; Caria, H.; Simões-Teixeira, H.; Aasen, T.; Nickel, R.; Jagger, D.J.; O’Neill, A.; Kelsell, D.P.; Fialho, G. A novel hearing loss-related mutation occurring in the GJB2 basal promoter. J. Med. Genet. 2007, 44, 721-725. [CrossRef]

38. Rodriguez-Paris, J.; Ballay, C.; Inserra, M.; Stidham, K.; Colen, T.; Roberson, J.; Gardner, P.; Schrijver, I. Genetic analysis of presbycusis by arrayed primer extension. Ann. Clin. Lab. Sci. 2008, 38, 352-360.

39. Fetoni, A.R.; Zorzi, V.; Paciello, F.; Ziraldo, G.; Peres, C.; Raspa, M.; Scavizzi, F.; Salvatore, A.M.; Crispino, G.; Tognola, G.; et al. Cx26 partial loss causes accelerated presbycusis by redox imbalance and dysregulation of Nfr2 pathway. Redox Biol. 2018, 19, 301-317. [CrossRef] [PubMed]

40. Nurk, S.; Koren, S.; Rhie, A.; Rautiainen, M.; Bzikadze, A.V.; Mikheenko, A.; Vollger, M.R.; Altemose, N.; Uralsky, L.; Gershman, A.; et al. The complete sequence of a human genome. bioRxiv 2021, 445798. [CrossRef]

41. Vollger, M.R.; Guitart, X.; Dishuck, P.C.; Mercuri, L.; Harvey, W.T.; Gershman, A.; Diekhans, M.; Sulovari, A.; Munson, K.M.; Lewis, A.M.; et al. Segmental duplications and their variation in a complete human genome. bioRxiv 2021, 445678. [CrossRef]

42. Fan, X.; Abbott, T.E.; Larson, D.; Chen, K. BreakDancer: Identification of genomic structural variation from paired-end read mapping. Curr. Protoc. Bioinform. 2014, 45, 15.6.1-15.6.11. [CrossRef]

43. Chen, K.; Wallis, J.W.; McLellan, M.D.; Larson, D.E.; Kalicki, J.M.; Pohl, C.S.; McGrath, S.D.; Wendl, M.C.; Zhang, Q.; Locke, D.P.; et al. BreakDancer: An algorithm for high-resolution mapping of genomic structural variation. Nat. Methods 2009, 6, 677-681. [CrossRef] [PubMed]

44. Becker, T.; Lee, W.P.; Leone, J.; Zhu, Q.; Zhang, C.; Liu, S.; Sargent, J.; Shanker, K.; Mil-homens, A.; Cerveira, E.; et al. FusorSV: An algorithm for optimally combining data from multiple structural variation detection methods. Genome Biol. 2018, 19, 38. [CrossRef] [PubMed] 